\title{
Desarrollo, consistencia interna, fiabilidad y validez de una escala de riesgos laborales en lengua española
}

\author{
Joan Boada-Grau ${ }^{*}$, Lluís Robert-Sentís ${ }^{1}$, Carme Gil-Ripoll ${ }^{2}$ y Andreu Vigil-Colet ${ }^{1}$ \\ ${ }^{1}$ Universitat Rovira i Virgili (CRAMC-Centre de Recerca de l'Avaluació i Mesura de la Conducta), Tarragona, España \\ ${ }^{2}$ Escuela de Alta Dirección y Administración, Barcelona, España
}

\begin{abstract}
Resumen: En el presente artículo estudiamos las propiedades psicométricas de una escala breve (RL-14) creada con el objetivo de detectar la información / formación recibida por los empleados, así como las patologías psicofisiológicas. Los participantes de la presente investigación son 1.489 empleados de diversos sectores como la formación, la construcción, el transporte, la sanidad, la industria, el comercio y la hostelería. Los resultados obtenidos demuestran, después de realizar análisis factorial tanto exploratorio como confirmatorio, una estructura constituida por dos factores: "(F1) Información y Formación” y “(F2) Patologías Psicofisiológicas”. Además, los dos factores obtenidos tienen una fiabilidad adecuada e igualmente se constatan indicios de validez de los dos factores si se toman como referencia algunas variables sociodemográficas, la fatiga, el burnout y la tensión laboral. En suma, la presente escala puede resultar idónea para identificar de manera apropiada los riesgos laborales. Futuras investigaciones podrían utilizar el RL-14 como una herramienta de screening en combinación con otros instrumentos.

Palabras clave: Riesgos laborales, patologías psicofisiológicas, información, formación, instrumento psicométrico.
\end{abstract}

\section{Introducción}

Los estados integrantes de la Unión Europea deben promover las mejoras del medio ambiente de trabajo con el fin de proteger la seguridad, la salud de los trabajadores y las condiciones laborales de los entornos de trabajo. Esto es lo que se desprende del Tratado de Roma (1997) y del Acta Única (1986). Así, la Unión Europea dictamina medidas jurídicas propias y promulga políticas específicas que vinculan a todos los estados miembros en su cumplimiento. En el ámbito de la salud laboral destacan dos directivas-marco: la de Higiene y Seguridad del Trabajo (80 / 1107 / CEE) que alude a la protección de los trabajadores contra los riesgos de exposición a agentes químicos físicos y biológicos durante el trabajo; y la de Seguridad y Salud Laboral (89 / 391 / CEE) sobre la aplicación de medidas para promover la mejora de la Seguridad de los empleados en el trabajo. Es más, la nueva estrategia de la Unión Europea, en el período 2007-2012, propone reducir de forma significativa la tasa global de accidentes de trabajo en la UE-27, indicando que ha de mejorar la protección de la salud y la seguridad de los empleados (Euroestat, 2009; European Comission, 2007).

En España, a partir de las conclusiones del National Survey on Safety and Health Enterprises Mangement (ENGE, 2009) deben desarrollarse tres aspectos: La evaluación de los riesgos, la formación de los empleados en seguridad y salud; y la prevención de los riesgos laborales. Y además, el Informe

* Dirección para correspondencia [Correspondence address]:

Joan Boada-Grau. Centre de Recerca d'Avaluació i Mesura de la Conducta (CRAMC). Departament de Psicologia. Universitat Rovira i Virgi-

li. Carretera de Valls, s/n. 43007 Tarragona (Catalunya, España).

E-mail: joan.boada@urv.cat; boadagrau@hotmail.com
Title: Development, internal consistency, reliability and validity of a scale of occupational hazards in Spanish.

Abstract: In this article, we study the psychometric properties of a short scale (RL-14) created in order to ascertain the information / training received by employees and their psychophysiological disorders. The participants of this study were 1.489 employees in various sectors including education, construction, transport, health, industry, trade and hospitality. The results of an exploratory and confirmatory factor analysis show a structure consisting of two factors: "(F1)Information and Training" and "(F2) Psychophysiological Pathologies". In addition, the two factors obtained have adequate reliability and present evidence of the validity of the two factors if some sociodemographic variables, such as fatigue, burnout and job strain are taken as benchmarks. In short, this scale may be suitable for the proper identification of workplace hazards. Future research could use the RL-14 as a tool for screening in combination with other instruments.

Keywords: Occupational hazards, psychophysiological pathologies, information, training, psychometric instrument.

de Estado sobre la Seguridad y la Salud Laboral, elaborado en 2011, indica la necesidad de desarrollar y consolidar la cultura de la prevención, insistir en la necesidad de información, potenciar la formación en materia de salud y prevención de riesgos laborales. En este sentido, las prácticas de recursos humanos (Boada-Grau y Gil-Ripoll, 2011) implementadas en las organizaciones pueden favorecer o dificultar las indicaciones mencionadas anteriormente.

Desde la Psicología de la Salud Laboral se pretende estudiar la etiología y la prevención de las enfermedades y daños a la salud desde el punto de vista de los factores organizacionales, sociales y psicológicos (Quick y Tetrick, 2003; Sauter y Hurrell, 1999). De forma complementaria, la Psicología de la Seguridad Laboral tiene como espacio característico el estudio de la etiología y la prevención de los accidentes laborales (Barling y Frone, 2004; López-Araújo y Osca, 2010; Sauter y Hurrell, 1999). Ambas tienen en común la aplicación de la psicología del bienestar y la calidad de vida laboral en las organizaciones, es por esto que las dos disciplinas científicas anteriormente descritas se integran en el concepto Psicología de la Seguridad y la Salud Laboral, esto viene avalado por el National Institute of Ocupational Safety and Health (NIOSH) de USA (Meliá, 2007).

De esta forma, Psicología de la Seguridad y la Salud Laboral (Barling y Frone, 2004; Geller, 1996; Houdmont y Leka, 2010; Leka y Houdmont, 2010; Quick, 1999; Quick y Tetrick, 2003; Sauter y Hurrell, 1999) abarca dos ámbitos interconectados, por un lado, el marco de la Seguridad que comprende la aplicación de los conocimientos de la psicología a la prevención de los accidentes laborales, las enfermedades profesionales y los daños y las patologías de la salud; por otro, el marco de la Salud que engloba los conoci- 
mientos de la psicología desplegados a la salud psicológica y orgánica de los empleados, analizando la incidencia de los riesgos psicosociales, químicos, mecánicos, etc.

En la Psicología de la Seguridad y la Salud Laboral existe un amplio abanico de metodologías de evaluación de los riesgos laborales, destacando entre ellos, los métodos de observación sistemática para identificar los riesgos potenciales en el laboratorio (Meliá, Sospedra y Rodrigo, 1993), la comunicación de los riesgos (Alavosius y Sulzer-Azaroff, 1990), los métodos cualitativos y cuantitativos de análisis (Meliá, 1999 y 2007; Peiró, 1999) y las escalas psicométricas (Boada-Grau, González, Vigil-Colet, Mañas y Agulló, 2009; Boada-Grau, Sánchez-García, Prizmic-Kuzmica y VigilColet, 2012; Líbano, Llorens, Salanova y Schaufeli, 2010; Meliá, 2004; Meliá y Becerril, 2007; Meliá y Sesé, 1999; Meliá et al., 1993; Ruberto, 2011).

Los temas propios de Psicología de la Seguridad y la Salud Laboral (Meliá, 2007), serían, sin ánimo de ser exhaustivos, entre otros, (1) los riesgos psicosociales como el estrés, el burnout y el mobbing; (2) las patologías psicológicas del trabajo como la depresión y la ansiedad; (3) las patologías físiológicas del trabajo como los trastornos musculares, esqueléticos, respiratorios, cardiovasculares y del sistema nervioso central; (4) los aspectos ergonómicos como el diseño de los puestos de trabajo y el manejo de herramientas; (5) los aspectos organizacionales y personales que afectan al trabajo como la formación, la satisfacción y la motivación; y (6) los procesos cognitivos como la forma de procesar la información recibida por los empleados. Es en este sentido, la escala (RL-14) que presentamos pretende evaluar algunos aspectos anteriormente enumerados.

En la Unión Europea, en el 2004, los riesgos psicosociales han sido identificados como unos de los riesgos emergentes y como una prioridad para su evaluación e intervención (EU-OSHA, 2007, 2010). Cox y Griffiths (1996) los definen como los aspectos del diseño del trabajo, de la organización y de la gestión del trabajo, y sus contextos sociales y ambientales, que tienen el potencial de causar daño psicológico, social y/o físico a los empleados. Así, como indican Leka, Jain, Zwetsloot y Cox (2010) es necesario que se aborden los riesgos psicosociales como una de las prioridades clave en el lugar de trabajo moderno con el fin de aumentar la sensibilización sobre dichos riesgos e incrementar las buenas prácticas con el fin de minimizar este tipo de riesgos laborales. Entre las principales patologías psicosociales más prevalentes encontramos el estrés laboral (De Croon, Sluiter, Blonk, Broersen y Frings-Dresen, 2004), la fatiga laboral (Machin y Hoare, 2008; Shadi, 2010), la tensión laboral (Strahan, Watson y Lennonb, 2008), los estados depresivos (Jolivet, 2010) y la ansiedad (Issever, Onen, Sabuncu y Altunkaynak, 2002), todas ellas, de etiología laboral.

Además, atendiendo a las indicaciones del Euroestat (2009) y de la European Comission (2007), que inciden en la necesidad de evaluar e intervenir, hemos considerado algunos tipos de patologías fisiológicas de distinta naturaleza originadas por el entorno laboral (Apparies, Riniolo y Porges,
1998; Machin y Hoare, 2008). En primer lugar, las musculares o esqueléticas (Robb y Mansfield, 2007) que hacen referencia a los dolores lumbares (Issever et al., 2002), a las artritis (Tang, 2010) y a los traumatismos (Wei, 2010). Segundo, las que afectan al sistema nervioso como las lesiones medulares (Burns, 2010) y las cefalalgias (Landy, 2011). Tercero, las que atañen al sistema respiratorio como el asma (Quirce, 2011), la apnea (Ruberto, 2011) y el enfisema (Santo-Tomás, 2011). Y finalmente, las patologías cardiovasculares (Chaparro, 2011; Krantz y McCeney, 2002) como la arteriosclerosis (Rosenström, 2011) y la hipertensión (Brison, 2000). Asímismo, también hemos tenido en cuenta la importancia de la información / formación siguiendo los preceptos del ENGE (2009) dado que es un aspecto relevante de la prevención de los riesgos laborales tal como ha quedado demostrado en investigaciones precedentes (Boada-Grau, De Diego y Agulló, 2004; Boada-Grau, De Diego y Macip, 2001).

Partiendo de los antecedentes expuestos y de la literatura revisada, hemos entendido que era necesario desarrollar un instrumento que permita evaluar la información / formación recibida y las patologías laborales tanto psicológicas como fisiológicas. Todo ello en la línea de lo que Dollard, Skinner, Tuckey y Bailey (2007) insisten, es decir, en la necesidad de crear y diseñar instrumentos de medida para evaluar los riesgos, que posibiliten establecer sistemas de vigilancia de los riesgos laborales con el fin de detectarlos de forma precoz.

Al hilo de lo anterior, el objetivo de este estudio es analizar la estructura interna (Análisis Factorial Exploratorio y Análisis Factorial Confirmatorio), la fiabilidad y la aportación de índices de validez convergente de la escala investigada. La presente investigación psicométrica, según Montero y León (2007), puede considerarse como un estudio instrumental. Finalmente, el RL-14, dado que es una versión breve (Lelito, Palumbo y Hanley, 2001; Tucker, Ogle, Davidson y Eilenberg, 1987), muestra algunas ventajas, entre otras, la presentación de una información inicial valiosa, la minimización del sesgo de respuesta relacionado con el cansancio de un instrumento de mayor longitud, la evaluación rápida y sencilla, y la reducción del tiempo dedicado a dar respuestas.

\section{Método}

\section{Participantes}

La muestra está compuesta por 1.489 empleados de España, de los que el $75.36 \%$ son hombres y el $24.64 \%$ son mujeres. Los entrevistados tienen una media de edad de 36.01 años (DT=12.20). Su Nivel Formación Académica se distribuye así: Sin estudios y Ningún certificado o título académico el 2.43\%; Estudios primarios inacabados y Certificado de escolaridad el 21.76\%; Bachiller elemental, FP-I o Graduado escolar el 41.60\%; Bachiller Superior, BUP, FPII, COU o PAU-Mayores 25 años el 18.66\%; Iniciando Estudios Universidad (Menos de 3 Cursos Completos Carrera) el 3.16\%; Tres Cursos Completos Carrera, Diplomatura o 
Ingeniero Técnico (1r ciclo) el 6.10\%; Licenciado, Ingeniero Superior o Arquitecto (2o ciclo) el 4.29\%; y Titulaciones universitarias de 3r Ciclo (Másters, Doctorado, etc.) el 2.0\%. La antigüedad media en el lugar de trabajo actual es de 7.99 años $(D T=9.34)$. La antigüedad media en su profesión es 12.55 años $(D T=11.88)$. Y la antigüedad media en su actual empresa es de 6.48 años $(D T=6.98)$. Los empleados desempeñan su trabajo en los siguientes sectores: Formación $(26.25 \%)$, construcción $(25.56 \%)$, transporte $(16.38 \%)$, sanidad $(12.42 \%)$, industria $(11.41 \%)$, comercio $(3.49 \%)$, hostelería $(2.10 \%)$ y otros $(2.39 \%)$.

\section{Instrumentos}

La escala RL-14 se desarrolló siguiendo el siguiente procedimiento. Un equipo de psicólogos y juristas con experiencia en prevención de riesgos laborales utilizaron técnicas grupales en empleados de distintos sectores socioeconómicos. Las técnicas empleadas fueron el brainstorming (Osborn, 1953; Canto, 2000; Gil, 2004) y el focus group (Morgan, 1998a; 1998b), a partir de los contenidos registrados en audio se construyeron un total de 26 ítems. Posteriormente, los análisis llevados a cabo sobre los mismos generaron la escala definitiva formada por 14 ítems. El formato de respuesta es un anclaje de cinco puntos $(1=$ nunca a $5=$ siempre).

La versión española del Maslach Burnout InventoryGeneral Survey (MBI-GS; Salanova, Schaufeli, Llorens, Peiró y Grau, 2000; Schaufeli, Leiter, Maslach y Jackson, 1996) consta de 15 ítems y tres subescalas. El anclaje de respuestas es de 6 puntos (desde "ninguna vez" a "todos los días"). La consistencia interna es .78 (eficacia profesional), .85 (cinismo) y .87 (agotamiento). La de eficacia profesional comprende 6 ítems (por ejemplo, "12.-He conseguido muchas cosas valiosas en este puesto"), la de cinismo tiene 5 ítems (por ejemplo, "9.-He perdido entusiasmo por mi trabajo") y la de agotamiento comprende 5 ítems (por ejemplo, “6.-Estoy 'quemado' por el trabajo").

La versión española del Swedish Occupational Fatigue Inventory (SOFI; Ahsberg, 2000; Ahsberg, Gamberale y Kjellberg, 1997; González, Moreno, Garrosa y López, 2005) evalúa la fatiga, tiene 15 ítems y cinco subescalas como: falta de energía ( 3 ítems; alfa = .82; por ejemplo "2.-Agotado"), cansancio físico ( 3 ítems; alfa $=.55$; por ejemplo " 9 .-Con calor"), disconfort físico ( 3 ítems; alfa $=.80$; por ejemplo "3.Entumecido"), falta de motivación (3 ítems; alfa $=.81$; por ejemplo "10.-Indiferente"), y somnolencia (3 ítems; alfa= .91; por ejemplo "11.-Bostezante"). Las respuestas se miden a través de un anclaje de 10 puntos (De " $1=$ Nada en absoluto" a " $10=$ En alto grado").

El Cuestionario de Tensión en el Trabajo (T3/15; Meliá, 1994) evalúa la medida de experiencia subjetiva de tensión asociada al desempeño del trabajo. La respuesta es dicotómica (si/no), la escala presenta una solución unifactorial. Consta de 15 ítems, por ejemplo, "2.-Estoy ansioso, tenso o preocupado a causa de mi trabajo" y "12.-Cuando no puedo terminar mi trabajo me pongo nervioso". Presenta una adecuada consistencia interna con una Alfa de Cronbach de .86.

Finalmente, para evaluar la validez convergente también se utilizaron algunos correlatos (Líbano et al., 2010) denominados indicadores externos (Gimeno, Benavides, Mira, Martínez y Benach, 2004) en forma de cuestiones que los informantes debían de contestar utilizando frecuencias. Así, se les hacían preguntas como la edad, la antigüedad (en el trabajo actual, en la profesión y en la empresa), las horas extras realizadas y las horas al año de formación (general y prevención de riesgos).

\section{Procedimiento}

Previo consentimiento de los responsables de las empresas y habiendo contactado con los empleados para participar en la investigación, las escalas se administraron en horario laboral e individualmente por parte de entrevistadores previamente entrenados a tal efecto. A los participantes se les dieron las instrucciones oportunas para contestar las escalas, asimismo se les aseguró la confidencialidad y el anonimato de los datos obtenidos.

\section{Análisis de Datos}

La muestra total de los 1.489 empleados fue dividida aleatoriamente en dos partes (744 y 745 respectivamente) con el fin de contribuir a la obtención de evidencias sobre la validez cruzada. En la primera submuestra (744 empleados) se efectuaron los análisis y descriptivos de los ítems y la dimensionalidad. El análisis factorial exploratorio (AFE) se efectuó con el método de extracción de ejes principales y aplicando la rotación oblimin; en este sentido, se han utilizado matrices de correlación policórica dado que están especialmente indicada en los casos en que los ítems presentan un formato de respuesta tipo Likert (Muthen y Kaplan, 1992). La utilización del programa FACTOR 7.2 (LorenzoSeva y Ferrando, 2006) para el análisis factorial exploratorio se debe a la posibilidad que ofrece, por un lado, realizar el análisis utilizando matrices de correlación policóricas y, por otro, proporciona el análisis paralelo que no está disponible en el SPSS 19.0 pero que sí ha sido utilizado para otros análisis. En la segunda submuestra (745 empleados), se realizó un análisis factorial confirmatorio (AFC) con el objetivo de validar la estructura factorial obtenida a partir de la primera submuestra. También, se calculó la fiabilidad mediante el alfa de Cronbach para cada uno de los dos factores que se extrajeron y, además, con el objetivo de evaluar la validez se han correlacionado los dos factores de la escala RL-14 con tres escalas (SOFI, MBI-GS y T3/15).

\section{Resultados}

Se efectuó un análisis factorial exploratorio (Tabla 1) mediante el programa FACTOR 7.2 (Lorenzo-Seva, 1999). Se obtuvo un índice Kaiser-Meyer-Olkin (KMO) de adecuación 
muestral de .88 lo cual demuestra que es adecuado para su factorización. El scree-test (Cattell, 1966) recomendó una solución de dos factores relacionados con la información/ formación y las patologías psicofisiológicas. También añadimos dos criterios para confirmar el número de factores a considerar, el análisis paralelo (Lattin, Carroll y Green, 2003) y el criterio "minimum average partial" de Vellicer (1976); esto criterios señalaron la adecuación de la solución de dos factores.

Obtenida la solución factorial más adecuada y con la finalidad de obtener una solución factorial más simple se utilizó el método de rotación Promin (Lorenzo-Seva, 1999), di- cho método de rotación oblicua tiende a obtener una solución lo más simple posible. A partir de los 26 ítems que se desarrollaron, se depuró la escala eliminando todos aquellos ítems que presentaran saturaciones inferiores a $.40 \mathrm{o}$ saturaciones complejas (superiores a .40 en más de un factor). Así, se descartaron 12 ítems y se escogieron 14 con las mayores saturaciones, de esta manera se han configurado dos factores de 9 y 5 ítems respectivamente. La varianza total de la escala es de $52.01 \%$ (Factor $1=35.48$ y Factor $2=16.52 \%$ ). Estos dos factores presentaron una correlación prácticamente nula (-.01) lo que implica que son independientes.

Tabla 1. Escala RL-14: Matriz de saturaciones de los factores extraídos del análisis factorial exploratorio y, además, la media y la desviación típica de los ítems.

\begin{tabular}{|c|c|c|c|c|}
\hline \multirow[t]{2}{*}{ Ítems } & \multicolumn{2}{|c|}{ Factores y Saturaciones } & \multicolumn{2}{|c|}{ Ítems } \\
\hline & F1 & $\mathrm{F} 2$ & Media & $D T$ \\
\hline 1.-Qué tipo de información recibe sobre los riesgos laborales que comporta su trabajo. & .83 & -.02 & 2.80 & 1.21 \\
\hline $\begin{array}{l}\text { 3.-Recibe Ud. información sobre los riesgos de su trabajo cuando se introduce nueva ma- } \\
\text { quinaria o nueva tecnología. }\end{array}$ & .77 & -.03 & 2.87 & 1.40 \\
\hline 5.-Cuando Ud. la solicita a su empresa, recibe información sobre los riesgos de su trabajo. & .70 & -.10 & 3.31 & 1.33 \\
\hline $\begin{array}{l}\text { 8.-La información que Ud. recibe sobre los riesgos de su trabajo es cuando la empresa con- } \\
\text { voca reuniones con esa finalidad. }\end{array}$ & .70 & .07 & 2.75 & 1.39 \\
\hline $\begin{array}{l}\text { 10.-La información que Ud. recibe sobre los riesgos de su trabajo es cuando los representan- } \\
\text { tes del personal se la comunican. }\end{array}$ & .58 & .08 & 2.71 & 1.36 \\
\hline $\begin{array}{l}\text { 13.-El empresario o el personal directivo le piden información sobre los riesgos laborales } \\
\text { que Ud. detecta en su trabajo. }\end{array}$ & .66 & .03 & 2.43 & 1.37 \\
\hline 6.-Recibe Ud. formación en materia de prevención de riesgos. & .84 & .01 & 2.57 & 1.34 \\
\hline 11.-El tiempo de formación de prevención de riesgos coincide con su jornada de trabajo. & .50 & .01 & 2.72 & 1.63 \\
\hline 14.-Recibe Ud. formación preventiva en materia de prevención de riesgos en la empresa. & .79 & .03 & 2.50 & 1.38 \\
\hline $\begin{array}{l}\text { 2.- Respecto a las patologías laborales musculares o esqueléticas como: Amputaciones, Artri- } \\
\text { tis ósea, Artritis reumatoide, Quemaduras, Anormalidad congénita, Disco cervical, Distrofia } \\
\text { muscular, Lesiones traumáticas, Espina bífida, Tumor cerebral, Trauma craneal, etc. Usted } \\
\text { ha padecido alguna debido a motivos laborales. }\end{array}$ & .01 & .53 & 1.60 & 1.01 \\
\hline $\begin{array}{l}\text { 4.-En cuanto a las patologías del sistema nervioso central como: Tumor cerebral, Trauma } \\
\text { craneal, Parálisis cerebral, Daño / trauma cerebro-vascular, Hemiplejia, Paraplejia, Tetraple- } \\
\text { jia, Cefalalgia o migraña, etc. Usted ha padecido alguna debido a motivos laborales. }\end{array}$ & .04 & .59 & 1.38 & .84 \\
\hline $\begin{array}{l}\text { 7.-Respecto a las patologías cardiovasculares como: Arritmias, Taquicardias, Lesión arterial } \\
\text { coronaria, Hipertensión, Defectos valvulares. Usted ha padecido alguna debido a motivos } \\
\text { laborales. }\end{array}$ & .01 & .73 & 1.28 & .74 \\
\hline $\begin{array}{l}\text { 9.-En cuanto a las patologías respiratorias como: Asma, Bronquitis crónica, Enfisema, } \\
\text { Neumocinosis, Silicosis, Rinitis, Neumonía intersticial. Usted ha padecido alguna debido a } \\
\text { motivos laborales. }\end{array}$ & -.03 & .59 & 1.25 & .73 \\
\hline $\begin{array}{l}\text { 12.-Respecto a las patologías psicológicas como: Estrés laboral, Depresión, Angustia, Ten- } \\
\text { sión. Usted ha padecido alguna debido a motivos laborales. }\end{array}$ & -.04 & .50 & 2.30 & 1.12 \\
\hline Varianza Explicada (52.01\%) & 35.48 & 16.52 & & \\
\hline
\end{tabular}

Varianza Explicada (52.01\%)

En la Tabla 2 se muestran la media, la desviación típica, la fiabilidad, los intervalos de confianza, los correlatos (edad, antigüedad, horas extras, etc.) y las correlaciones entre los dos factores de la escala RL-14 y los factores de las escalas SOFI, MBI-GS y T3/15. Las correlaciones del instrumento que presentamos con las tres escalas anteriores y algunos criterios externos informan de algunos índices de validez. En este sentido, se puede observar algunas correlaciones significativas como las que se describen. Así, el primer factor que expresa información y formación correlaciona positivamente con las horas al año de formación general y las horas al año en formación de prevención de riesgos, y de forma negativa con días al año de baja laboral, cinismo (MBI-GS) y tensión laboral (T3/15). Y el segundo factor que hace referencia a las patologías psicofisiológicas presenta varias correlaciones, una de ellas negativa como la eficacia (MBI-GS) y catorce de positivas como: correlatos externos (edad, antigüedad, horas extras y baja laboral), fatiga laboral (falta de energía, cansancio físico, disconfort físico, falta de motivación y somnolencia), burnout (agotamiento y cinismo) y tensión laboral. 
Tabla 2. Factores de la escala RL-14: Estadísticos descriptivos, fiabilidad, intervalos de confianza y correlaciones con las variables sociodemográficas y los factores de las escalas (SOFI, MBI-GS y T3/15), y matriz de correlaciones entre los dos factores de la escala analizada.

\begin{tabular}{|c|c|c|}
\hline & F1.-Información y Formación. & F2.-Patologías Psicofisiológicas \\
\hline Media & 24.70 & 7.84 \\
\hline$D T$ & 9.17 & 3.01 \\
\hline Fiabilidad & .89 & .68 \\
\hline Intervalo de Confianza & $.88-.90$ & $.65-.70$ \\
\hline Edad & .04 & $.06^{*}$ \\
\hline Antigüedad en el trabajo actual & .01 & $.08^{* *}$ \\
\hline Antigüedad en la profesión & .02 & $.11^{* *}$ \\
\hline Antigüedad en la empresa & .03 & $.10^{* *}$ \\
\hline Horas extras & .01 & $.12^{* *}$ \\
\hline Días/año de ILT & $-.08 * *$ & $.08^{* *}$ \\
\hline Horas/año de formación general & $.11^{* *}$ & -.01 \\
\hline Horas/año de formación en PRL & $.12^{* *}$ & .01 \\
\hline SOFI (Falta de Energía) & .04 & $.28^{* *}$ \\
\hline SOFI (Cansancio Físico) & .02 & $.25^{* *}$ \\
\hline SOFI (Disconfort Físico) & .01 & $.29 * *$ \\
\hline SOFI (Falta de Motivación) & .01 & $.27 * *$ \\
\hline SOFI (Somnolencia) & -.07 & $.24 * *$ \\
\hline MBI-GS (Agotamiento) & .06 & $.37 *$ \\
\hline MBI-GS (Cinismo) & $-.12^{*}$ & $.28^{* *}$ \\
\hline MBI-GS (Eficacia) & .01 & $-.15^{*}$ \\
\hline T3/15 (Tensión Laboral) & $-.08^{* *}$ & $.35^{* *}$ \\
\hline F1.-Información y Formación. & --- & --- \\
\hline F2.-Patologías Psicofisiológicas. & -.01 & --- \\
\hline
\end{tabular}
** $\mathrm{p}<.01 ; * \mathrm{p}<.05$

Dada la necesidad de confirmar la estructura del AFE, se efectuó un análisis factorial confirmatorio con el Mplus VERSIÓN 5.1 y el método de estimación fué promin. Los índices son: el error cuadrático medio de aproximación (RMSEA $\leq .06)$, el índice de ajuste comparado $(\mathrm{CFI} \geq .95) \mathrm{y}$ el índice de Tucker-Lewis (TLI $\geq .95$ ). Los resultados obtenidos presentan un buen ajuste del modelo dado que los índices (RMSEA $=.06$; CFI $=.95$ y TLI $=.94$ ) se situan cerca de los valores considerados óptimos. Los coeficientes estandarizados se presentan en la Figura 1.

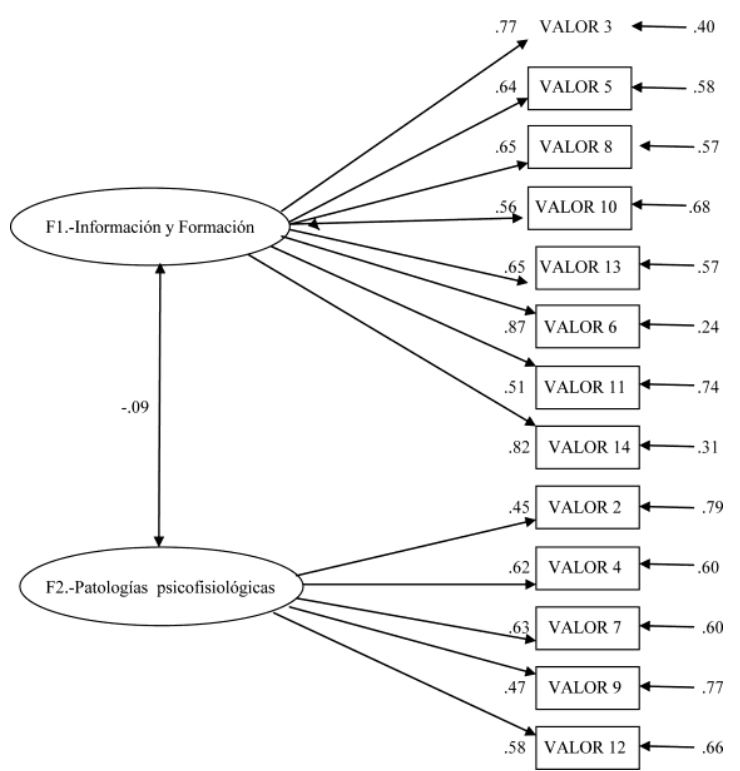

Figura 1. Análisis factorial confirmatorio de la escala RL-14 (Chi-square = $297.980 ; \mathrm{df}=76 ; \mathrm{P}$-value $=.0000 ;$ RMSEA $=.06 ; \mathrm{CFI}=.95$ y $\mathrm{TLI}=.94)$.

\section{Discusión y Conclusiones}

Los resultados de la presente investigación no pueden ser comparadas con otras dado que la escala que presentamos es de elaboración propia. Los resultados respaldan que la escala analizada, sobre riesgos laborales, posee una estructura interna de dos factores, una fiabilidad adecuada y, además, unos índices de validez apropiados. El presente instrumento ha sido validado empíricamente y tiene unas propiedades psicométricas óptimas, además ofrece la posibilidad de evaluar los riesgos laborales en diversos sectores productivos.

Los resultados del análisis factorial exploratorio indica la existencia de dos factores que permiten explorar los riesgos laborales. El primer factor, lo denominamos "Información y Formación", el cual alude a la información que recibe el empleado tanto sobre sus riesgos laborales en general y cuando se introducen nuevas herramientas de trabajo, como quién se lo comunica (el mismo empresario, los directivos y/o los representantes). Además, también hace referencia a la formación específica en riesgos laborales recibida. Explica el $35.48 \%$ de la varianza y está integrado por nueve ítems (números: 1, 3, 5, 6, 8, 10, 11, 13 y 14). La fiabilidad del presente factor es .89 con un intervalo de confianza entre .88 y .90 .

Por otro lado, el segundo factor "Patologías psicofisiológicas" hace referencia a un conjunto de dolencias y/o enfermedades de etiología profesional como las musculoesqueléticas (amputaciones, artritis, quemaduras, lesiones traumáticas, etc.), las del sistema nervioso central (daño cerebro-vascular, cefalalgia o migraña, etc.), las cardiovasculares (arritmias, taquicardias, hipertensión, etc.), las respirato- 
rias (bronquitis, enfisema, rinitis, neumonía, etc.) y las psicológicas (estrés laboral, depresión, angustia, tensión, etc.). La fiabilidad de este factor es .68 con un intervalo de confianza entre .65 y .70. Este está conformado por cinco ítems con los números 2, 4, 7, 9 y 12 que explican el 16.52 $\%$ de la varianza. Además, la correlación entre F1 y F2 indica una muy baja asociación (-.01).

Igualmente, los resultados del análisis factorial confirmatorio de la presente escala apoyan el modelo de los dos factores y muestra un ajuste satisfactorio. Todo ello se corrobora mediante los índice obtenidos dado que presentan un buen ajuste del modelo $(\mathrm{RMSEA}=.06$; $\mathrm{CFI}=.95 \mathrm{y}$ TLI $=$ .94). Las saturaciones de los ítems oscilan entre .45 y .87 .

En cuanto a la validez, se refleja que la presente escala obtiene correlaciones significativas con otros correlatos y algunas escalas de contraste. Se observa que el factor "1.Información y formación" se asocia algunas variables como las bajas anuales y la formación, el cinismo y la tensión laboral; y el segundo factor "2.-Patologías psicofisiológicas" se asocia a algunos criterios externos (edad, antigüedad, etc.), a la fatiga, al burnout y a la tensión laboral.

En conclusión, los distintos análisis realizados han aportado evidencia de la existencia de una estructura bifactorial: "1.-Información y Formación” y "2.-Patologías psicofisiológicas", a la vez que muestra unos índices estadísticos adecuados (Tabachnick y Fidell, 2007). La escala RL-14 se configura como un instrumento breve, de rápida aplicación e interpretación; y de fácil comprensión; a través de las dos subescalas que lo conforman, posibilita una valoración independiente de cada una de ellas. La primera de ellas permite la información sobre los riesgos laborales de su trabajo, así como la recibe, es decir, si es a través de su empresa y/o representantes sindicales; y, además, si recibe formación específica sobre la prevención de los riesgos laborales. La segunda evalúa diversos tipos de patologías como las músculoesqueléticas, las del sistema nervioso central, las cardiovasculares, las respiratorias y las psicológicas.

En cuanto a la aplicabilidad, la información obtenida de la evaluación realizada a través del RL-14 puede permitir la implantación de programas de prevención (Machin y Hoare, 2008). Así, dado que la escala presenta buenas propiedades psicométricas abastece a los profesionales prevencionistas de un instrumento con el que obtener datos destinados a crear programas preventivos en vigilancia de la salud. Ésta se entiende como la recogida, el análisis y la interpretación sistemática de los datos referidos a la salud laboral de los trabajadores con la finalidad de proteger su salud y de prevenirlos de la enfermedad (Boix et al., 2000).

A modo de ejemplo, la presente escala puede ser considerada como un criterio de valoración en la vigilancia de la salud basada en la evidencia (Boada-Grau et al., 2004; Boada-Grau, De Diego, Agulló y Mañas, 2005; Verbeek, Van Dijk, Mailmivaraa, Hulshof y Räsänen, 2002), de esta forma puede ayudar a la toma de decisiones en la prevención de la salud sustentada en pruebas científicas contrastadas que cumplan criterios psicométricos óptimos (Boada-Grau et al., 2009; Boada-Grau et al., 2011; Spencer, Robertson y Folkard, 2006), todo ello facilita la recogida, el análisis y la interpretación de los datos obtenidos con la finalidad tanto de proteger la salud laboral como de prevenir los posibles riesgos laborales originados (Thacker y Berkelman, 1992), por ejemplo, como una herramienta de "screening" que pueden utilizar los técnicos de prevención. Es más, en el marco del control de la gestión preventiva (Organización Internacional del Trabajo, 2001b), la escala RL-14 viene a cumplir un requisito importante de aquella como es el de facilitar la dimensión cuantitativa de la medida en contraposición a la cualitativa tan mayoritaria en este ámbito.

Al hilo de lo anterior, confiamos que la RL-14 sea utilizada como una herramienta para el diseño de intervenciones dirigidas aumentar la calidad de vida laboral de los empleados (Comisión Nacional de Seguridad y Salud en el Trabajo, 2007; Organización Internacional del Trabajo, 2001a). Los tipos de intervenciones (Boada-Grau, 1999) donde podría ser utilizada la presente escala son la primaria (dirigida a eliminar los riesgos), por ejemplo, cuando se incorpora un nuevo trabajador en la organización; la secundaria (que persigue reducir la incidencia y prevalencia de los riesgos), por ejemplo cuando un trabajador cambia de un puesto de trabajo a otro; y la terciaria (orientada a paliar los efectos negativos y la destructividad derivada de los riesgos), por ejemplo, después de producirse un incidente laboral. Un ejemplo de intervención psicosocial serían los Programas de Ayuda al Empleado (Solé y Balduque, 2006) los cuales pueden facilitar información valiosa, por ejemplo, cuando se realizan las horas de formación anuales, con el fin de evitar situaciones de alto riesgo laboral en el presente y en el futuro.

El origen de las investigaciones que pretendemos efectuar en el futuro, serán las limitaciones del actual. Éstas las comentamos a continuación. Primera, sería oportuno analizar el funcionamiento de la escala en diferentes colectivos de trabajadores inmigrantes como los de origen africano, latinoamericano, centroeuropeo, etc. dado que provienen de culturas laborales en las que los aspectos relacionados con la prevención de los riesgos están menos desarrolladas. Segunda, sería preciso analizar la validez discriminante (Blanch y Aluja, 2009) atendiendo tanto el tipo de trabajo ("cuello azul" vs "cuello blanco") como el origen diverso (por ejemplo, africano, latinoamericano y centroeuropeo). Tercera, la validación de un instrumento es un proceso dinámico el cual no acaba con el diseño y publicación del mismo, así pues, es previsible que nuevas investigaciones aporten nuevos datos sobre el mismo (Padilla, Gómez, Hidalgo y Muñiz, 2006). Y cuarta, consideramos que sería necesario analizar si algunos aspectos de la personalidad de los empleados, como la impulsividad, el atrevimiento, la minuciosidad, la agresividad y el neuroticismo podrían relacionarse con el procesamiento de la información recibida, la formación realizada y las patologías psicofisiológicas. 


\section{Referencias}

Alavosius, M. P. y Sulzer-Azaroff, B. (1990). Acquisition and maintenance of health-care routines as a function of feedback density. Journal of Applied behaviour Analysis, 23, 151-162.

Apparies R.J., Riniolo, T.C. y Porges, S.W. (1998). A psychophysiological investigation of the effects of driving longer-combination vehicles. Ergonomics, 41, 581- 592.

Ahsberg, E. (2000). Dimensions of fatigue in different working populations. Scandinavian Journal of Psychology 41, 231-241.

Ahsberg, E., Gamberale, F. y Kjellberg, A. (1997). Perceived quality of fatigue during different occupational tasks. Development of a questionnaire. International Journal of Industrial Ergonomics 20, 121-135.

Barling, J. y Frone, M. (2004). The Psychology of Workplace Safety. Washington DC: American Psychological Association.

Blanch, A. y Aluja, A. (2009).Validation study of the Spanish Version of the Work-Family Conflict Questionnaire (CCTF). The Spanish Journal of Psychology, 12, 746-755.

Boada-Grau, J. (1999). Psicología del (Trabajo + Organizaciones + Recursos Humanos). Barcelona: PPU Ediciones.

Boada-Grau, J., De Diego, R. y Agulló, E. (2004). Burnout and psychosomatics manifestations as consequences of organizational climate and labour motivation. Psicothema, 16, 125-131.

Boada-Grau, J., De Diego, R., Agulló, E. y Mañas, M.A. (2005). Absenteeism from work as consequent of organizational variables. Psicothema, 17, 212-218.

Boada-Grau, J., De Diego, R. y Macip, S. (2001). Cultura organizacional y formación contínua: Incidencia en la Prevención de Riesgos Laborales. Revista de Psicología del Trabajo y de las Organizaciones, 17, 91-107.

Boada-Grau, J. y Gil-Ripoll, C. (2011). Measure of human resource management practices: psychometric properties and factorial structure of the questionnaire PRH-33. Anales de Psicologia, 27, 527-535.

Boada-Grau, J., González, S., Vigil-Colet, A., Mañas, M.A. y Agulló, S. (2009). CONS-32: Development of a laboral risk prevention questionnaire for the construction industry. Psicothema, 21, 165-169.

Boada-Grau, J., Sánchez-García, J.C., Prizmic-Kuzmica, A-J. y Vigil-Colet, A. (2012). Work health and hygiene in the transport industry (TRANS18): Factorial structure, reliability and validity. The Spanish Journal of Psychology, 15, 357-366.

Boix, P., Benavides, F.G., Soriano, G., Moreno, N., Roe, J.M. y GarcíaGómez, M. (2000). Criterios básicos para la vigilancia de la salud de los trabajadores: Decálogo sobre la vigilancia de la salud en el trabajo. Archivo de Prevención de Riesgos Laborales, 3, 175-177.

Brison, C. (2000). Women, work and cardiovascular disease. Occupational Medicine: State of the Art Reviews, 15, 49-57.

Burns, S.M (2010). Psychosocial predictors of employment status among men living with spinal cord injury. Rehabilitation Psychology, 55, 90-95.

Canto, J. M. (2000). Dinámica de Grupos: Aspectos técnicos, ámbitos de intervención y fundamentos teóricos. Málaga: Aljibe.

Cattell, R. B. (1966). The scree test for the number of factors. Multivariate Behavioral Research, 1, 245-276.

Chaparro, M.A. (2011). High cardiovascular risk in Spanish workers. Nutrition Matabolism and Cardiovascular Diseases, 21, 231-236.

Comisión Nacional de Seguridad y Salud en el Trabajo (2007). Estrategia española de seguridad y salud en el trabajo (2007-2012).Madrid: Ministerio de Trabajo e Inmigración - Instituto Nacional de Seguridad e Higiene en el Trabajo.

Cox, T. y Griffiths, A. (1996). The assessment of psychosocial hazards at work. In M.J. Schabracq, J.A.M. Winnubst, y C.L. Cooper (Eds.), Handbook of work and health psychology (pp. 127-146). Chichester: Wiley.

De Croon, E.M., Sluiter, J.K., Blonk, R.W. B., Broersen, J.P.J. y FringsDresen, M.H.W. (2004). Stressful Work, Psychological Job Strain, and Turnover: A 2-Year. Prospective Cohort Study of Truck Drivers. Journal of Applied Psychology, 89, 442-454.

Dollard, M., Skinner, N., Tuckey, M.R. y Bailey, T. (2007). National surveillance of psychosocial risk factors in the workplace: An international overview. Work \& Stress, 21, 1-29.
ENGE (2009). Conclusions from National Survey on Safety and Health Enterprises Mangement. Madrid: Instituto Nacional de Seguridad e Higiene en el Trabajo.

EU-OSHA. (2007). Expert forecast on emerging psychosocial risks related to occupational safety and health, European Risk Observatory Report. Luxembourg: Office for Official Publications of the European Communities.

EU-OSHA. (2010). European enterprise survey on new and emerging risks, European Risk Observatory Report. Luxembourg: Office for Official Publications of the European Communities.

Euroestat (2009). Population and social conditions. Luxembourg: Publications Office of the European Union.

European Comission (2007). Improving quality and productivity at work: Community strategy 2007-2012 on health and safety at work (2007-2012). EU: Bruselas.

Geller, E.S. (1996). The psychology of safety: How to improve behaviors and attitudes on the job. Radnor, PA: Chilton Book Company.

Gil, F. (2004). Técnicas para generar ideas y para solucionar problemas.En F. Gil y C. M. Alcover (Coord.). Técnicas grupales en contextos organizacionales (pp.145-170). Madrid: Pirámide

Gimeno, D., Benavides, F.G., Mira, M., Martínez, J.M., y Benach, J. (2004). External Validation of Psychological Jobs Demands in a Bus Drives Sample. Journal of Occupational Health, 26, 43-48.

González, J.L., Moreno, B., Garrosa, E. y López, A. (2005). Spanish version of the Swedish Occupational Fatigue Inventory (SOFI): Factorial replication, reliability and validity. International Journal of Industrial Ergonomics, 35, 737-746.

Houdmont, J. y Leka, S. (Eds.) (2010). Contemporary occupational health psychology: Global perspectives in research and practice. Chichester: Wiley-Blackwell.

Issever, H., Onen, L., Sabuncu, H. H., y Altunkaynak, O. (2002). Personality characteristics, psychological symptoms and anxiety levels of drivers in charge of urban transportation in Istanbul. Occupational Medicine, 52, 297-303.

Jolivet, A. (2010). Linking hospital workers' organizational work environment to depressive symptoms: A mediating effect of effort-reward imbalance? The ORSOSA study. Social Science y Medicine, 71, 540-545.

Krantz, D. S., y McCeney, M. K. (2002). Effects of psychological and social factors on organic disease: A critical reassessment of research on coronary heart disease. Annual Review of Psychology, 53, 341-369.

Landy, S.H. (2011). Assessing the impact of migraine onset on work productivity. Journal of Occupational and Environmental Medicine / American College of Occupational And Environmental Medicine, 53, 74-81.

Lattin, J., Carroll, D. J. y Green, P. E. (2003). Analyzing multivariate data. Pacific Grove. Duxbury Press.

Leka, S. y Houdmont, J. (Eds.) (2010).Occupational health psychology. Chichester: Wiley-Blackwell.

Leka, S., Jain, A., Zwetsloot, G. y Cox, T. (2010). Policy-level interventions and work-related psychosocial risk management in the European Union. Work \& Stress, 24, 298-307.

Lelito, R.H., Palumbo, L.O. y Hanley, M. (2001). Psychometric evaluation of a brief geriatric depression screen. Aging Ment Health, 5, 387-93.

Líbano, M., Llorens, S., Salanova, M. y Schaufeli, W. (2010). Validity of a brief workaholism scale. Psicothema, 22, 143-150.

López-Araújo, B. y Osca, A. (2010). Influencia de algunas variables organizacionales sobre la salud y la accidentabilidad laboral. Anales de Psicología, 26, 89-94.

Lorenzo-Seva, U. (1999). Promin: a method for oblique factor rotation. Multivariate Behavioral Research, 34, 347-365.

Lorenzo-Seva, U., y Ferrando, P.J. (2006). FACTOR: A computer program to fit the exploratory factor analysis model. Behavioral Research Methods, Instruments and Computers, 38, 88-91.

Machin, M. A. y Hoare P.N. (2008). The role of workload and driver coping styles in predicting bus drivers' need for recovery, positive and negative affect, and physical symptoms. Anxiety, Stress, \& Coping, 21, 359-375.

Machin, M. A. y Hoare P.N. (2008). The role of workload and driver coping styles in predicting bus drivers' need for recovery, positive and negative affect, and physical symptoms. Anxiety, Stress, y Coping, 21, 359-375. 
Meliá, J. L. (1994) La medición de la tensión en el trabajo. Revista de Psicología del Trabajo y Organizaciones, 10, 17-38.

Meliá, J. L. (1999). Medición y métodos de intervención en psicología de la seguridad y prevención de accidentes. Revista de Psicología del Trabajo y de las Organizaciones, 15, 237-266.

Meliá, J. L. (2004). La Batería Valenciana PREVACC de la Universidad de Valencia: La evaluación de las dimensiones comportamentales, grupales y organizacionales que afectan a los accidentes laborales. Trabajo presentado al Tercer Congreso Internacional de Prevención de Riesgos Laborales. Santiago de Compostela.

Meliá, J. L. y Becerril, M. (2007). Fuentes psicosociales de estrés y burnout en el sector de la construcción: un modelo de ecuaciones. Psicothema, 19, 679-686.

Meliá, J. L. y Sessé, A (1999). La medida del clima de seguridad y salud laboral. Anales de Psicología, 15, 269-289.

Meliá, J. L., Sospedra, M. J. y Rodrigo, M. F. (1993). Una segunda replicación del estudio del Cuestionario para la medida del Riesgo Basal (RB 3/17): Fiabilidad, Validez, Estructura Factorial y Análisis Diferenciales en una muestra de sujetos accidentados. Revista de Psicología del Trabajo y de las Organizaciones, 9, 251-263.

Meliá, J.L. (2007). El factor humano en la seguridad laboral. Psicología de la seguridad y la salud laboral. Madrid: Lettera.

Montero, I. y León, O. G. (2007). A guide for naming research studies in Psychology. International Journal of Clinical and Health Psychology, 7, 847862

Morgan, D. L. (1998a). The Focus Group Guidebook. Focus Group Kit 1. Thousand Oaks, Ca: Sage.

Morgan, D.L. (1998b). Planning Focues Group. Focus Group Kit 2. Thousand Oaks, Ca: Sage.

Muthen, B. y Kaplan, D. (1992). A comparison of some methodologies for the factor analysis of non-normal Likert variables: A note on the size of the model. British Journal of Mathematical and Statistical Psychology, 45, 1930 .

Organización Internacional del Trabajo (2001a). Enciclopedia de Salud y Seguridad en el Trabajo. Ginebra: Oficina Internacional del Trabajo.

Organización Internacional del Trabajo (2001b). Proyecto de directrices técnicas de la OIT sobre sistemas de gestión de la seguridad y salud en el trabajo. Ginebra: Oficina Internacional del Trabajo.

Osborn, A. F. (1953). Imaginación aplicada. Principios y procedimientos para pensar creando.Madrid: Verflex.

Padilla, J.L., Gómez, J., Hidalgo, M.D. y Muñiz, J. (2006). La evaluación de las consecuencias del uso de los tests en la teoría de la validez. Psicothema, 18, 307-312.

Peiró, J. M. (1999).Valoración de riesgos psicosociales y estrategias de prevención: el modelo "AMIGO" como base de la metodología "Prevenilab/Psicosocial". Revista de Psicología del Trabajo y de las Organizaciones, 15, 267-314.

Quick, J.C. y Tetrick, L.E (2003). Handbook of Occupational Health Psychology. Washington DC: American Psychological Association.

Quick, J.C. (1999). Occupational health psychology: Historical roots and future directions. Health Psychology, 18, 82-88.
Quirce, S. (2011). New causes of occupational asthma. Current Opinion In Allergy And Clinical Immunology, 11, 80-85.

Robb, M. J. M. y Mansfield, N. J. (2007). Self-reported musculoskeletal problems amongst professional truck drivers. Ergonomics, 50, $814-827$.

Rosenström, T. (2011) Change in job strain and progression of atherosclerosis. Journal of Occupational Health Psychology, 16, 201-210.

Ruberto, M. (2011). Obstructive Sleep Apnoea Syndrome (OSAS) and mood disorders in a population of public transport drivers. Medicina del Lavoro, 102, 201-207.

Salanova, M., Schaufeli, W.B., Llorens, S., Peiró, J.M., y Grau, R. (2000). Desde el 'burnout' al 'engagement': cuna nueva perspectiva?. Revista de Psicología del Trabajo y las Organizaciones, 16, 117-134.

Santo-Tomás, L.H. (2011). Emphysema and chronic obstructive pulmonary disease in coal miners. Current Opinion In Pulmonary Medicine, 17, 123-128.

Sauter, S. L. y Hurrell, J.J. (1999).Occupational Health Psychology: Origins, Content, and Direction. Professional Psychology: Research and Practice, 30, 117-122.

Schaufeli, W.B., Leiter, M.P., Maslach, C. y Jackson, S.E. (1996). Maslach Burnout Inventory - General Survey. En C. Mas1ach, S.E. Jackson y M.P. Leiter (Eds.). The Maslach Burnout Inventory- Test Manual (3rd ed.) Palo Alto, CA: Consulting Psychologists Press.

Shadi, J.W. (2010). Type of employment and occupational health in urban public transport in the city of Kinshasa. Sante Publique, 22, 647-656.

Solé, M. D. y Balduque, M. (2006). El programa de ayuda al empleado (EAP): in tervención individual en la prevención de riesgos psicosociales. Madrid: Instituto Nacional de Seguridad e Higiene en el Trabajo.

Spencer, M. B., Robertson, K. A. y Folkard, S. (2006). The development of a fatigue/ risk index for shiftworkers (Research Rep. 446). Sudbury, England: HSE Books.

Strahan, C., Watson, B. y Lennonb, A. (2008). Can organisational safety climate and occupational stress predict work-related driver fatigue? Transportation Research part F-Traffic Psychology and Behaviour, 11, 418-426.

Tabachnick, B.G., y Fidell, L.S. (2007). Using Multivariate Statistic. Boston: Allyn and Bacon.

Tang, K. (2010). The Work Instability Scale for Rheumatoid Arthritis (RAWIS): Does it work in osteoarthritis? An International Journal of Quality of Life Aspects of Treatment, Care y Rehabilitation, 19, 1068-1075.

Thacker, S. B. y Berkelman, R. (1992). History of public health surveillance. En W.Halperin y E.L. Barker (Ed.). Public Health Surveillance. (pp.62-75). New York: Van Nostrand Reinhold, Co.

Tucker, M.A, Ogle, S. J., Davidson, J. G. y Eilenberg, M.D. (1987). Validation of a brief screening test for depression in the elderly. Age Ageing, 16, 139-144.

Vellicer, W. F. (1976). Determining the number of components from the matrix of partial correlations. Psychometrike, 41, 321-327.

Verbeek, J.H., Van Dijk, F.J., Mailmivaraa, A., Hulshof, C. y Räsänen, K. (2002). Evidence-based medicine for occupational health. Scandinavian Journal of Work Environment Health, 28, 197-204.

Wei, W. (2010). Work-related mild-moderate traumatic brain injuries due to falls. Brain Injury, 24, 1363-1371.

(Artículo recibido: 14-10-2011, revisión: 25-01-2012, aceptado: 06-02-2012) 